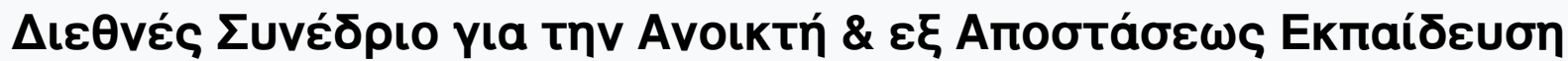

Tón. 6, Ap. 1A (2011)

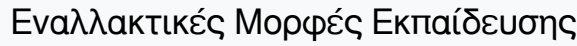

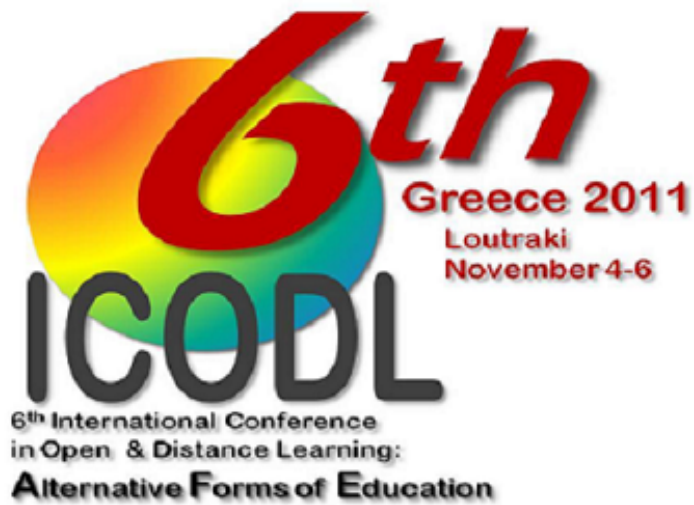

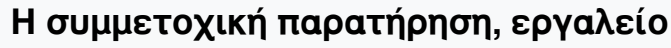

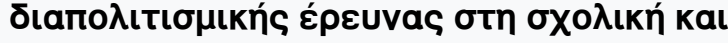

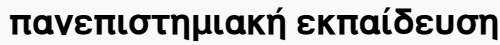

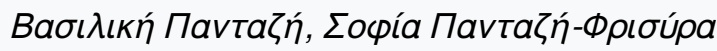
doi: $10.12681 /$ icodl. 690

TOMOEA

PART/MEPOE A 


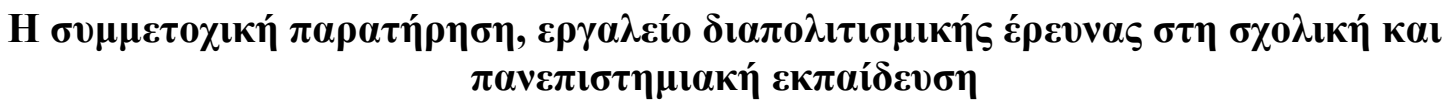

\title{
Participant observation, a tool for intercultural research in school and university education
}

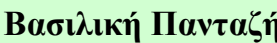

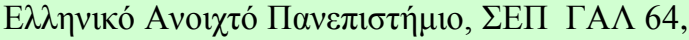
vaspant@yahoo.gr

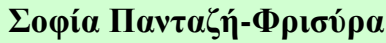

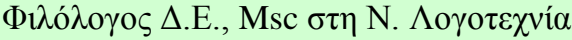

sophiapantazi@gmail.com

\begin{abstract}
A multitude of research conducted in the recent years has been employing participant observation as a tool, thus implementing a principally sociological and ethnological methodology in educational environments, and that with intercultural features.

Within intercultural environments, the researcher- observer is to be seen as the protagonist of an interaction among individuals- bearers of different cultural identities, who experiences the special relationship with the difference he/ she is trying to interpret, grasp, accept and familiarise themselves with.

To the researcher-observer, the meeting with intercultural group members constitutes the starting point, the initiation of the process of reaching one another, which can even result in a cultural observer- observed interbreeding, thus confirming one of the features of intercultural communication, namely mutual influence.

Within the framework of the present lecture we will try to indicate the main problems of participant observation concerning

1. The risk of influencing the identity references of the observer

2. The observer's objectivity

3. The typicality of the study cases.

Moreover, we will seek to show that if the results of research conducted in intercultural environments are duly exploited in formal education, they could trigger the use of groundbreaking educational tools, based on the principles of interculturality.
\end{abstract}

\section{Пєрі́ $\eta \psi \eta$}

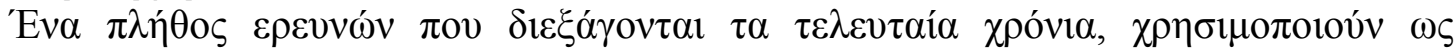

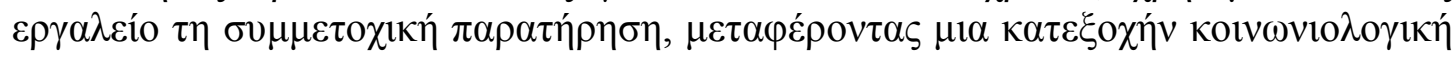

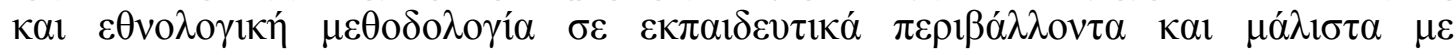

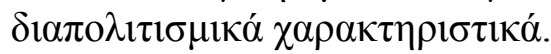

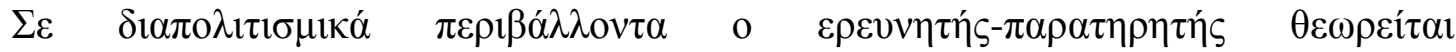

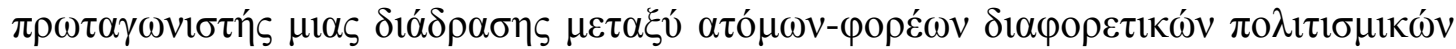

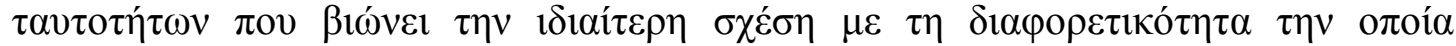

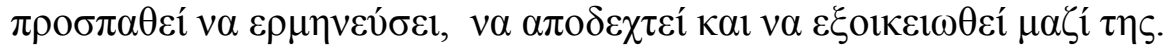

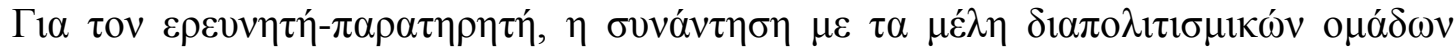

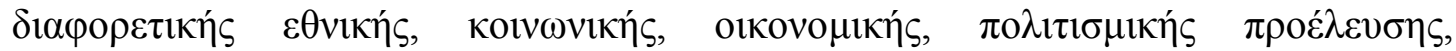

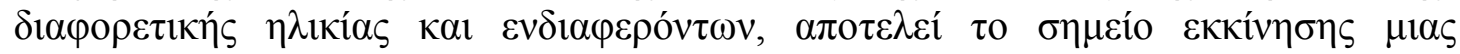

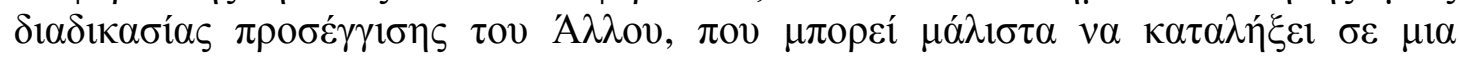




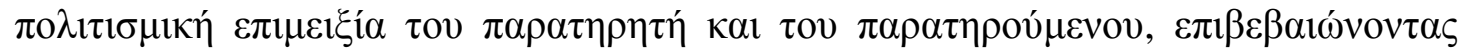

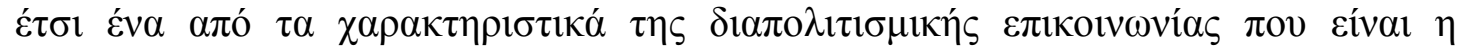

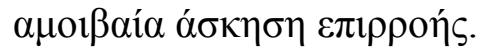

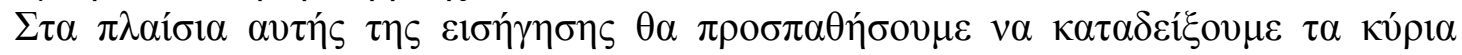

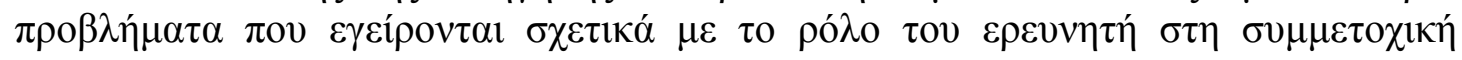

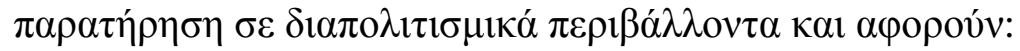

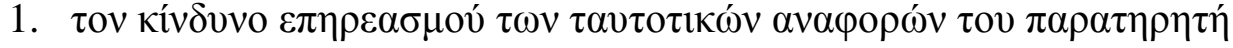

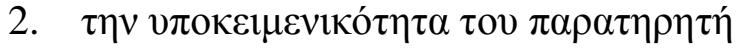

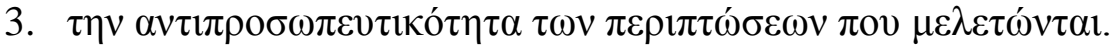

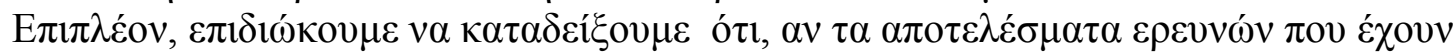

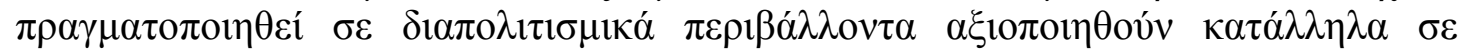

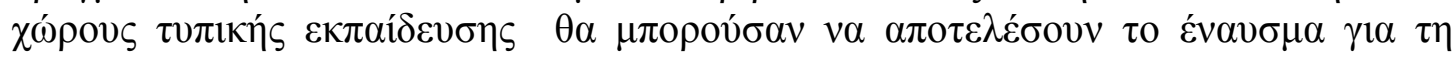

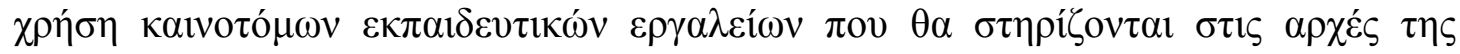

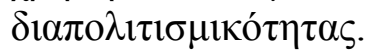

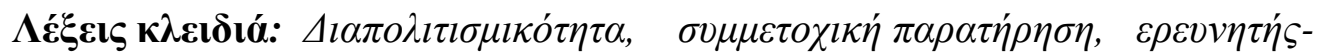
$\pi \alpha \rho \alpha \tau \eta \eta \tau \eta \dot{\varsigma, ~ \kappa \alpha l v o \tau o ́ \mu \alpha ~ \varepsilon \kappa \pi \alpha l \delta \varepsilon v \tau \imath \kappa \alpha ́ ~ \varepsilon \rho \gamma \alpha \lambda \varepsilon i ́ \alpha ~}$

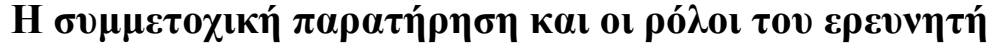

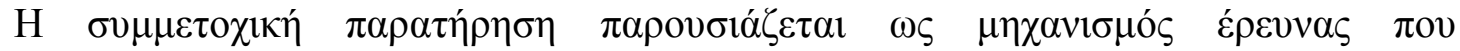

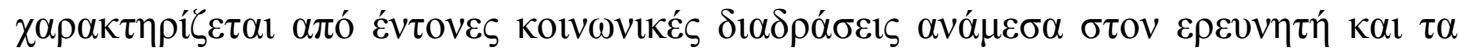

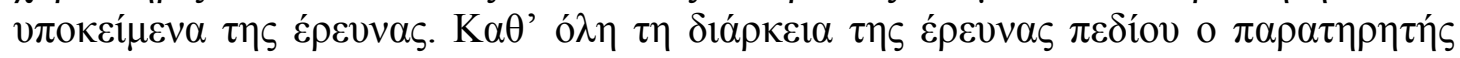

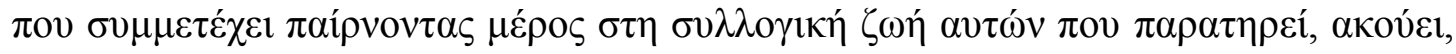

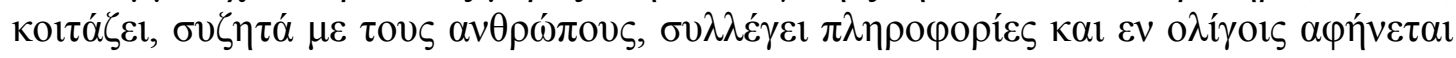

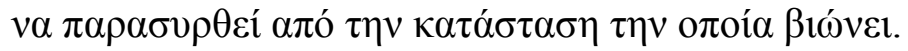

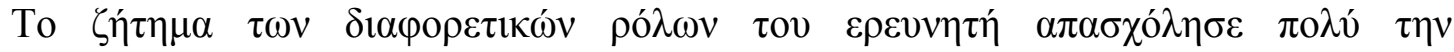

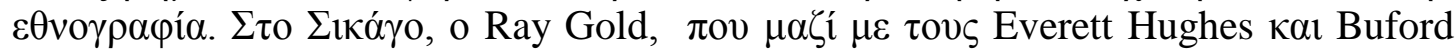

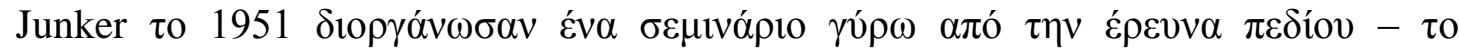

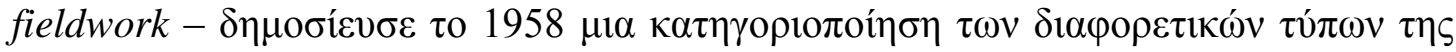

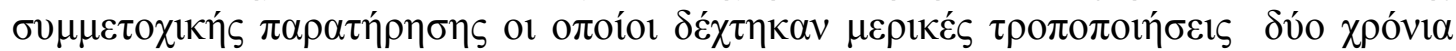

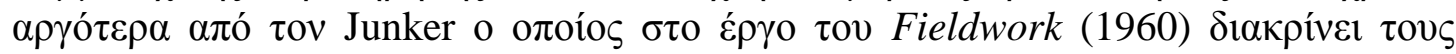

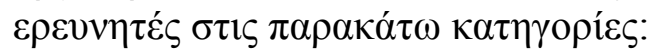

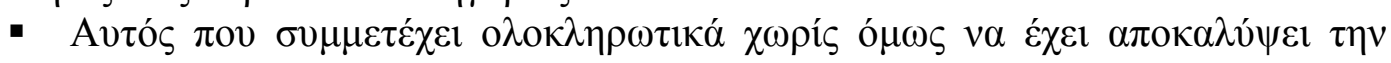

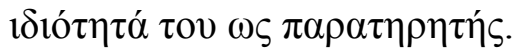

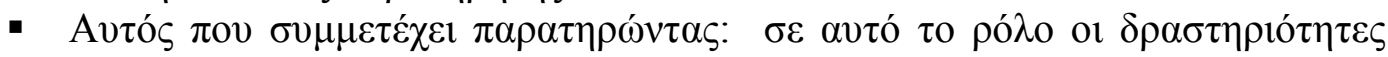

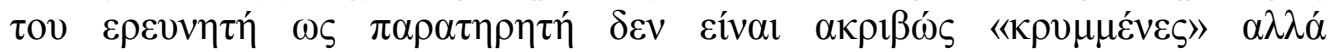

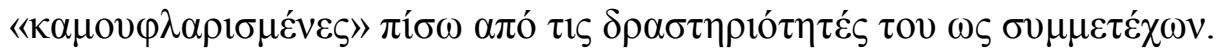

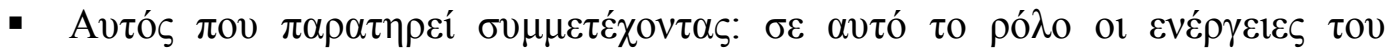

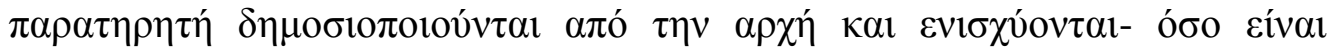

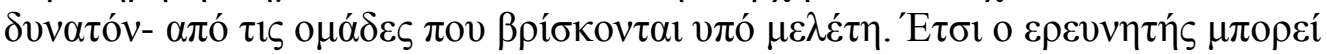

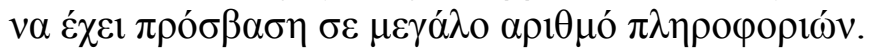

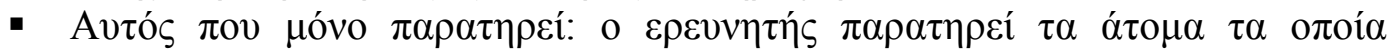

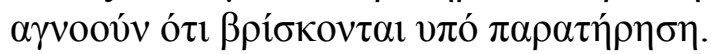

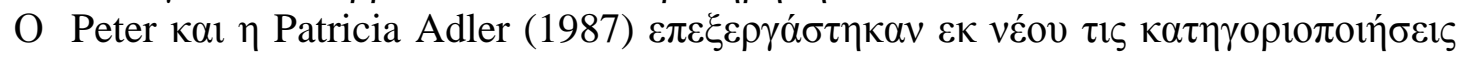

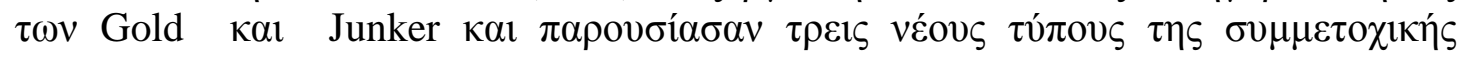
$\pi \alpha \rho \alpha \tau \eta \dot{\rho \eta \sigma \eta \varsigma: ~}$

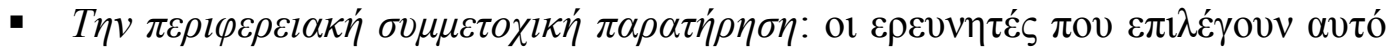

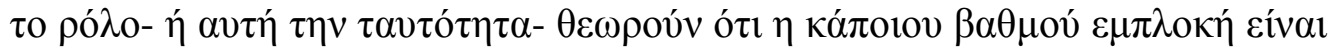

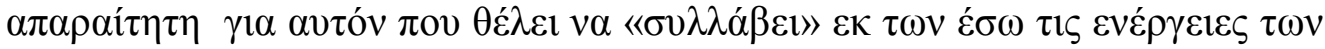

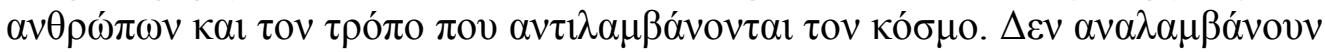




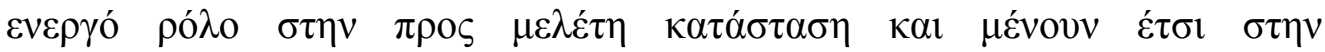

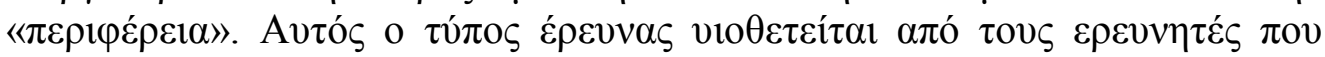

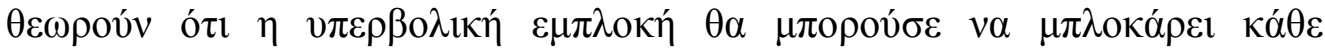

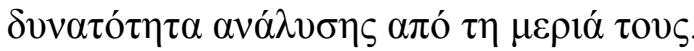

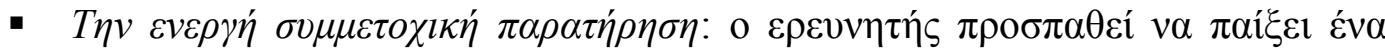

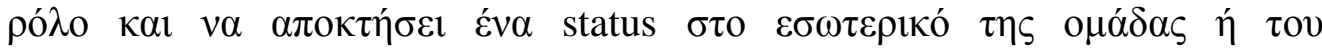

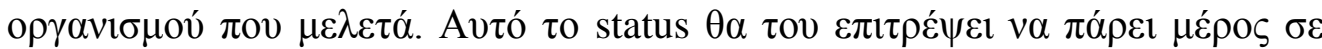

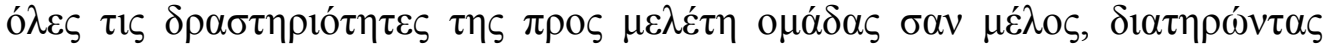

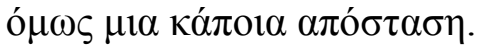

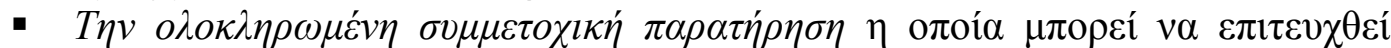

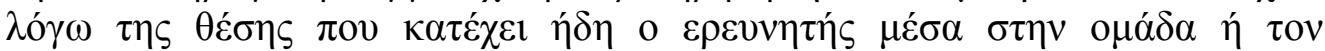

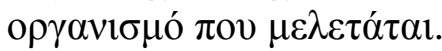

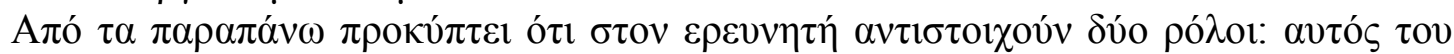

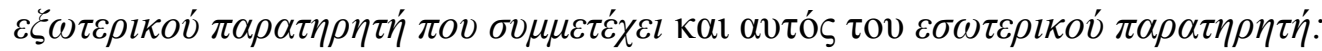

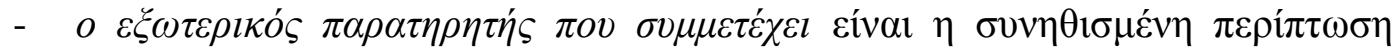

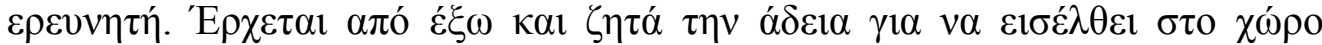

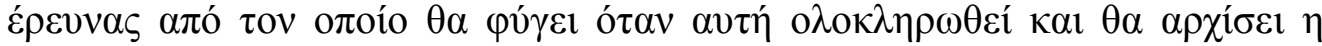

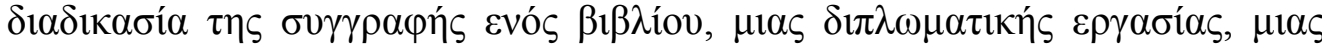

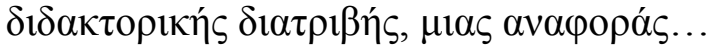

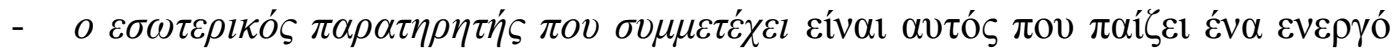

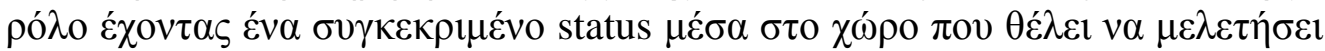

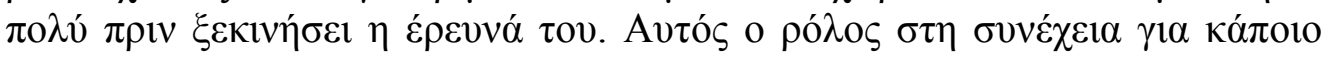

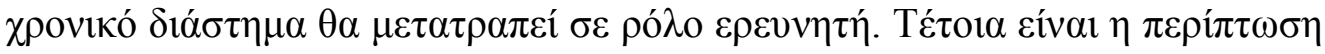

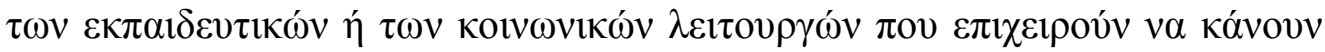

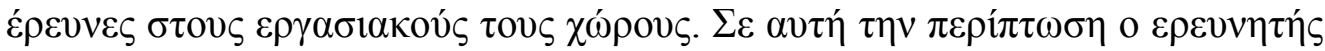

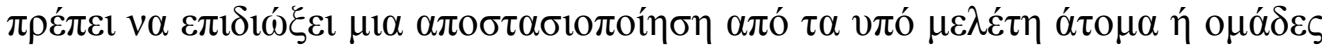

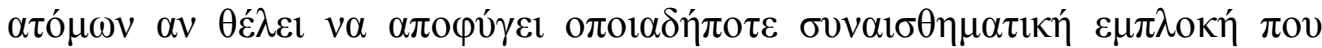

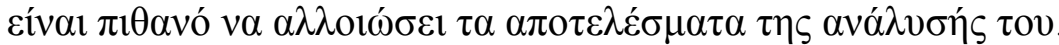

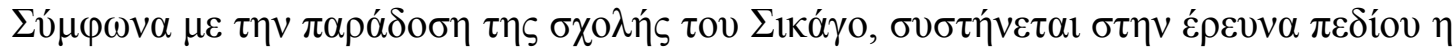

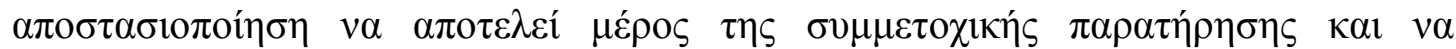

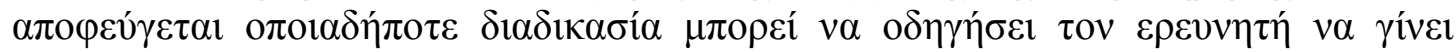

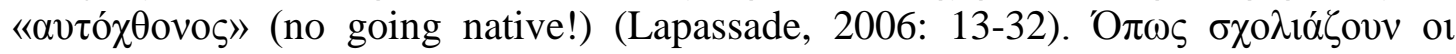

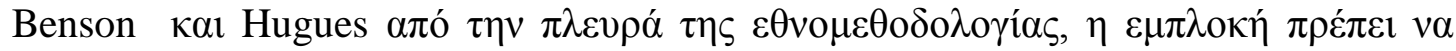

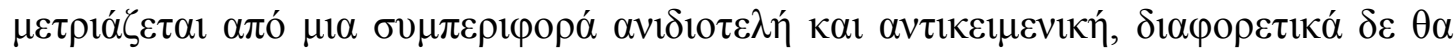

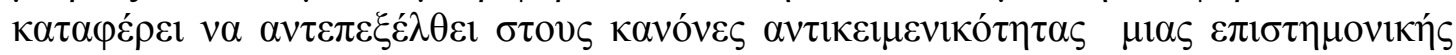

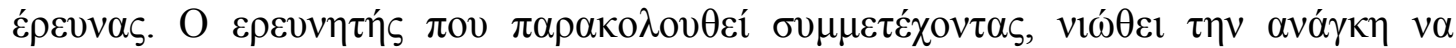

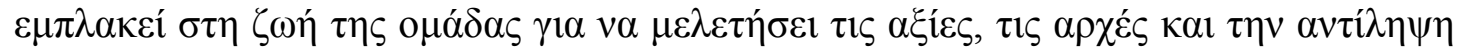

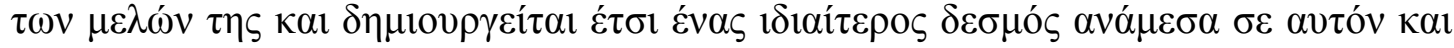

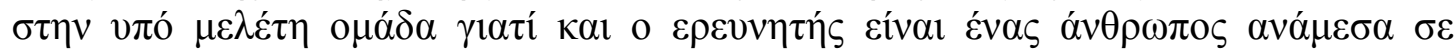

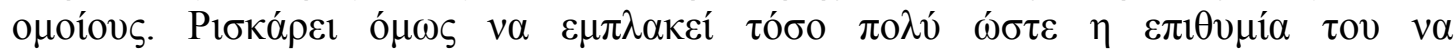

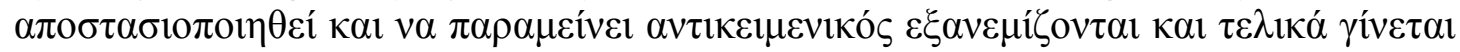

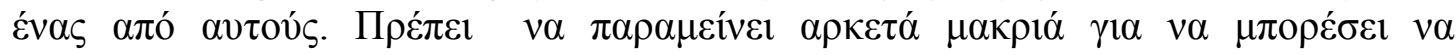

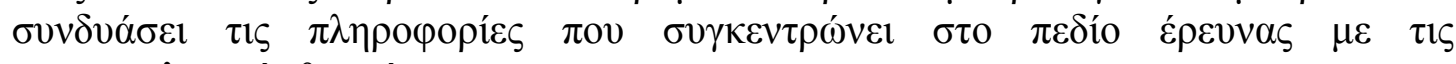

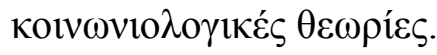

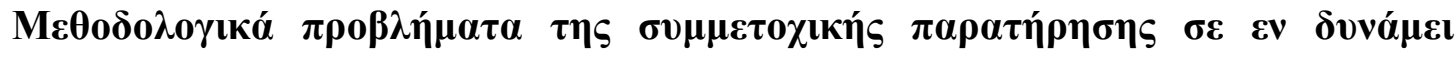

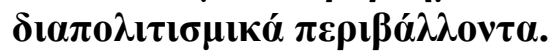

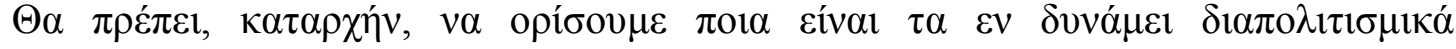

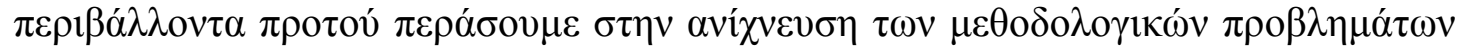

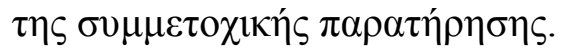




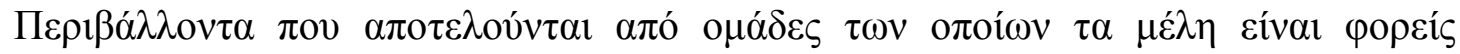

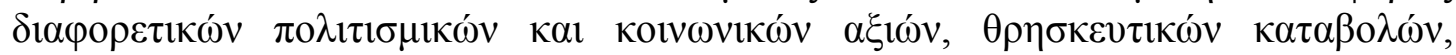

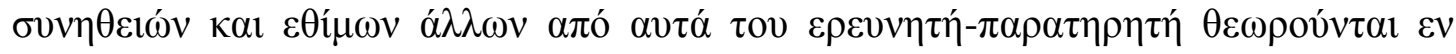

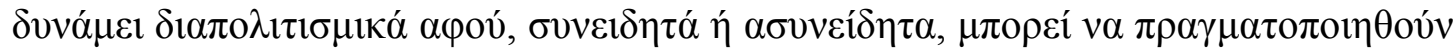

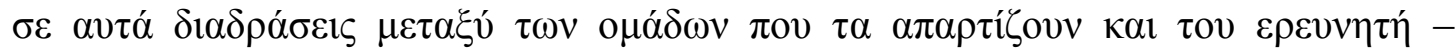

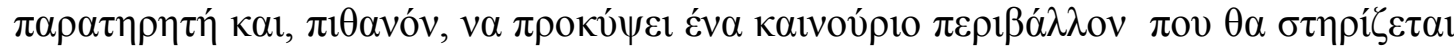

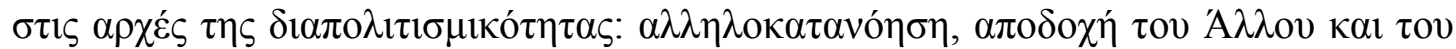

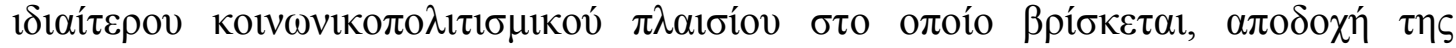

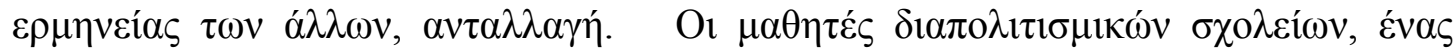

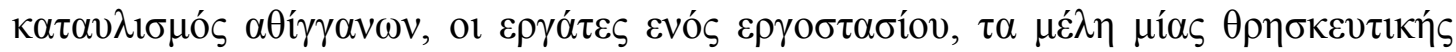

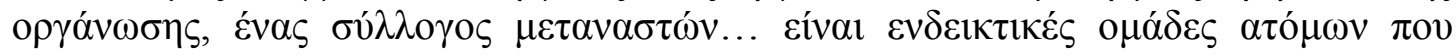

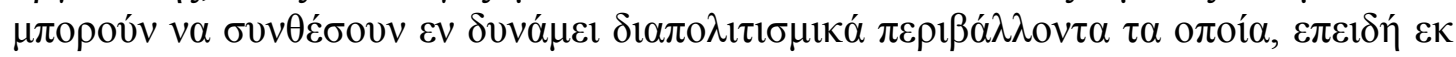

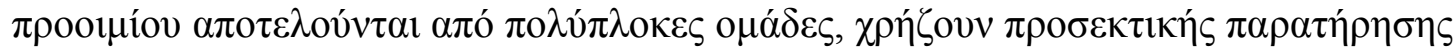

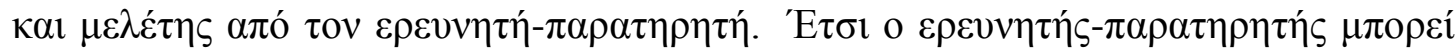

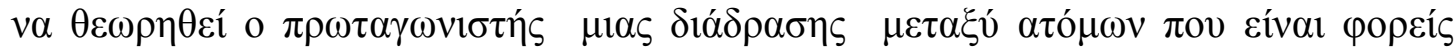

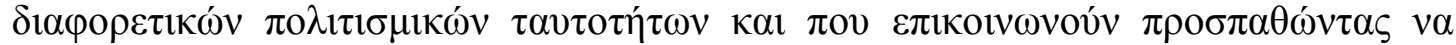

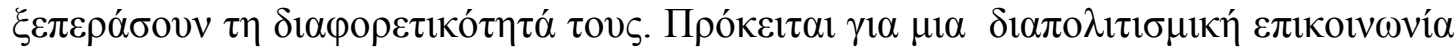

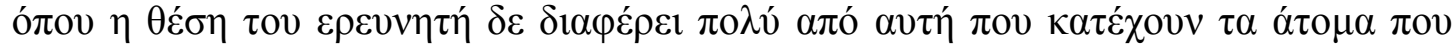

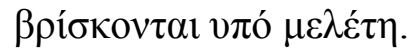

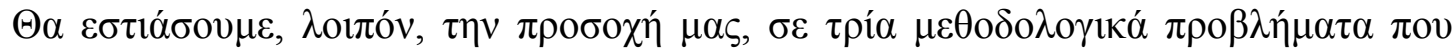

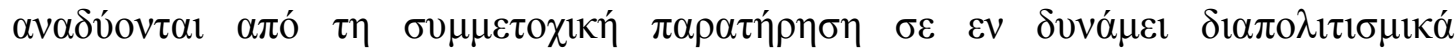
$\pi \varepsilon \rho 1 \beta \alpha \dot{\lambda} \lambda \mathrm{ov} \tau \alpha$ :

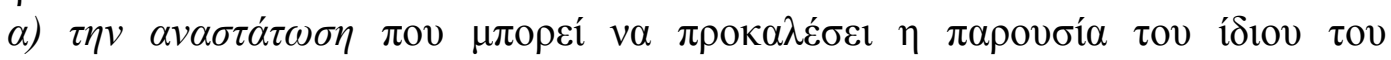

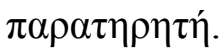

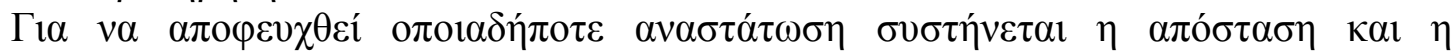

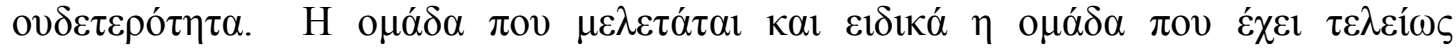

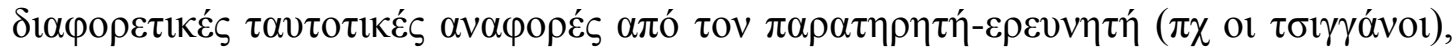

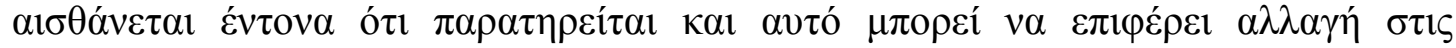

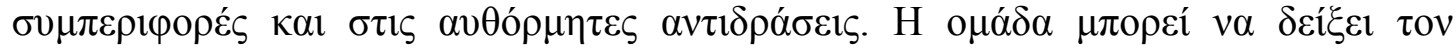

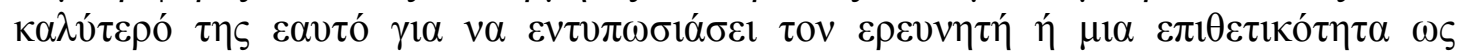

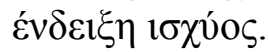

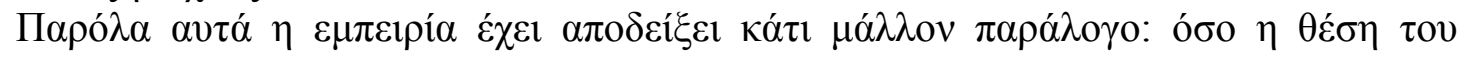

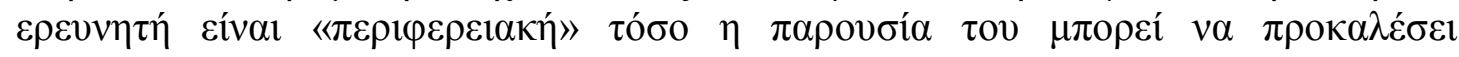

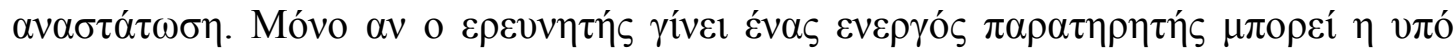

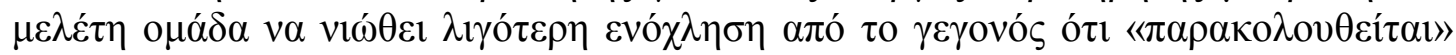

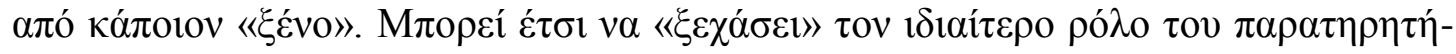

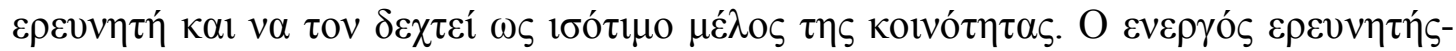

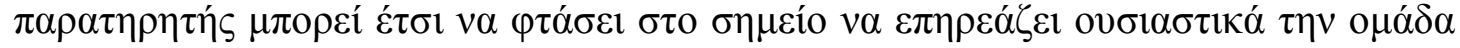

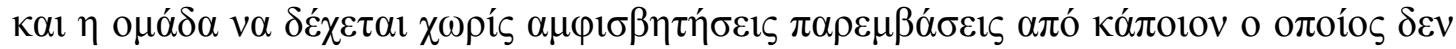

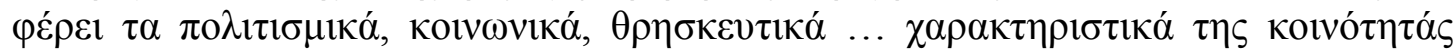

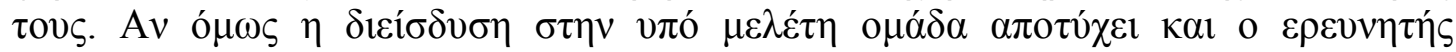

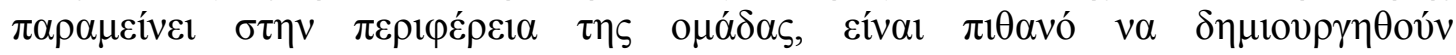

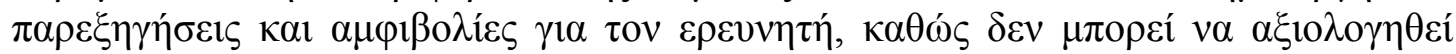

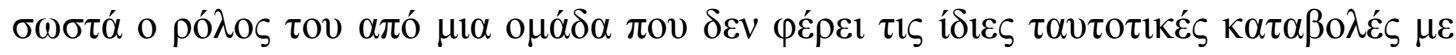
eкeívov.

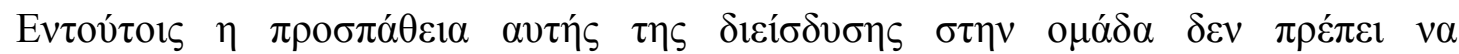

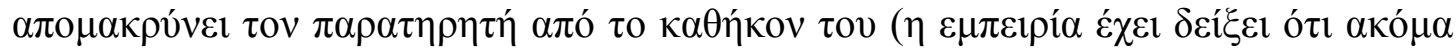

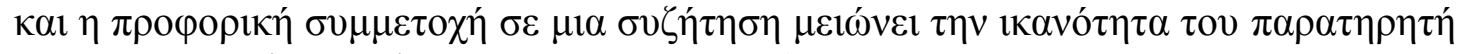

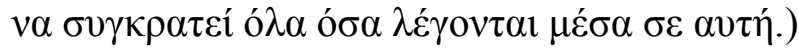




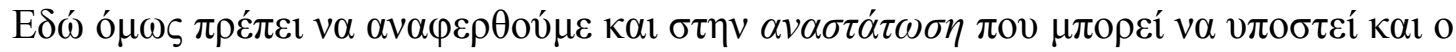

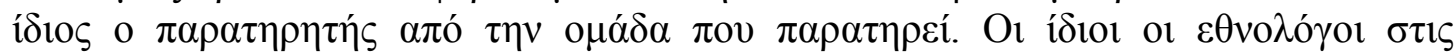

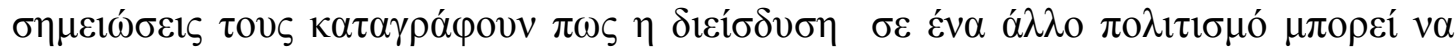

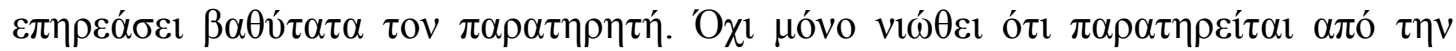

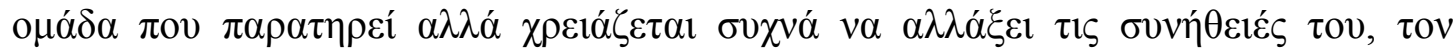

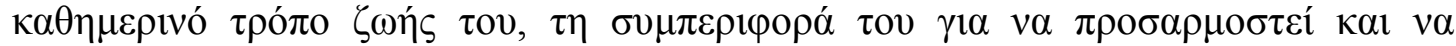

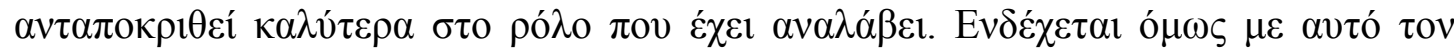

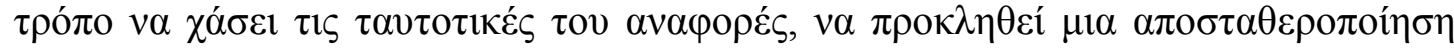

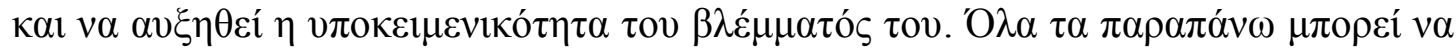

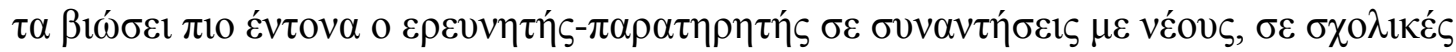

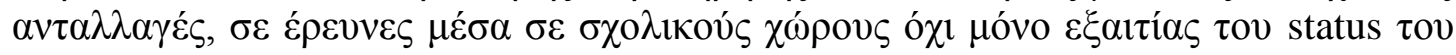

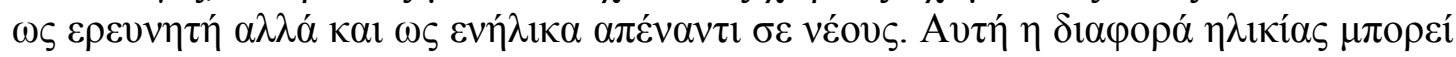

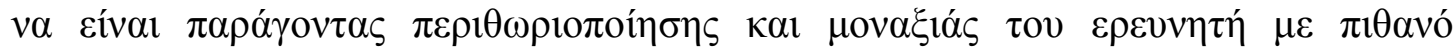

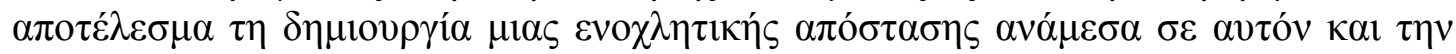
vлó $\mu \varepsilon \lambda \dot{\varepsilon} \tau \eta ~ o \mu \alpha ́ \delta \alpha$.

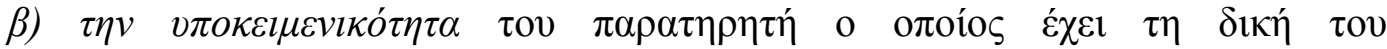

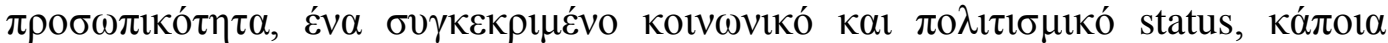

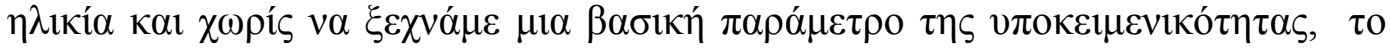
pú̀o.

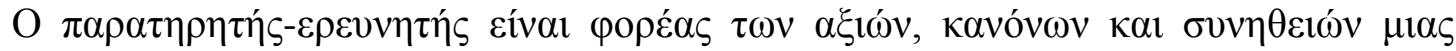

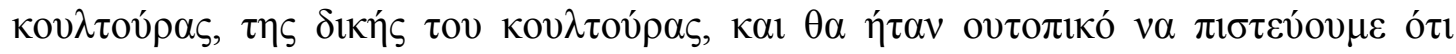

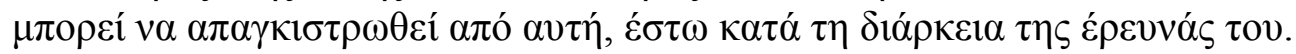

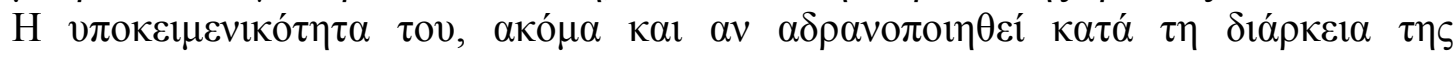

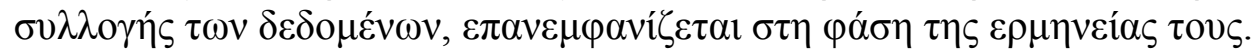

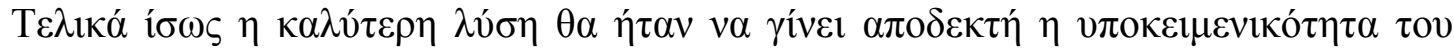

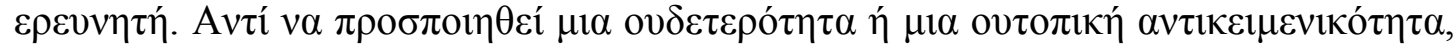

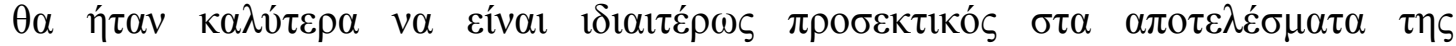

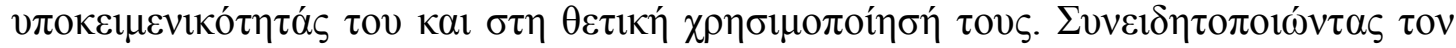

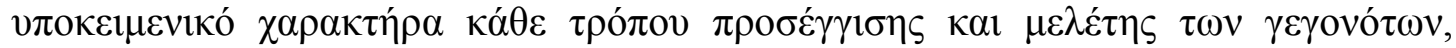

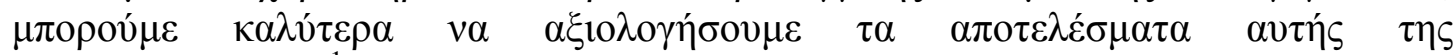

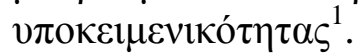

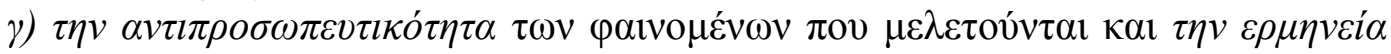

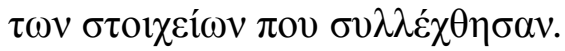

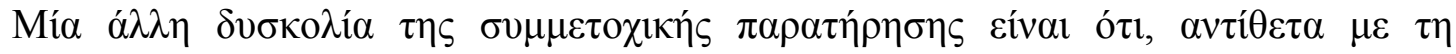

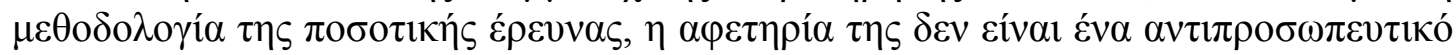

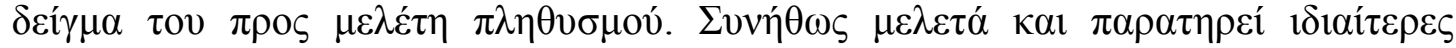

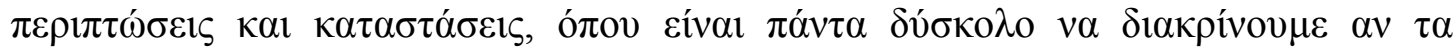

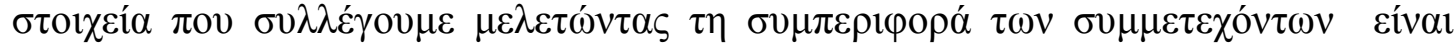

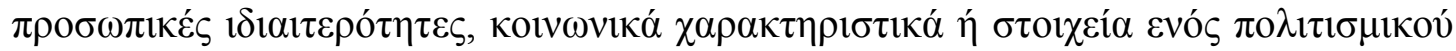

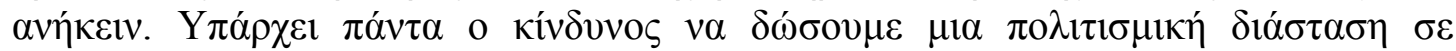

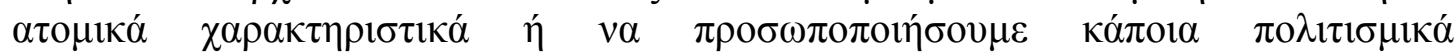

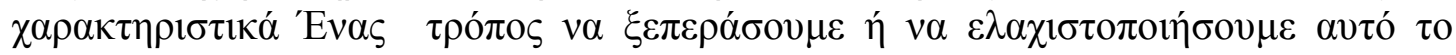

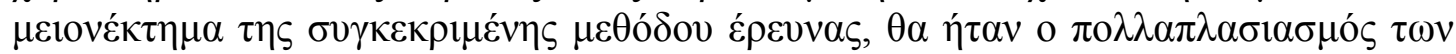

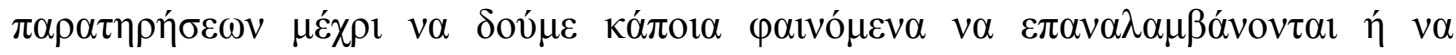

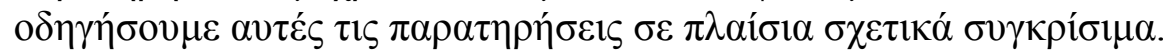

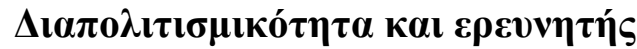

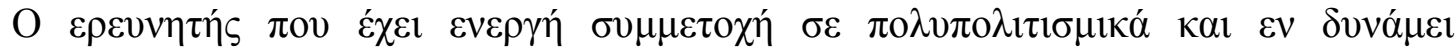

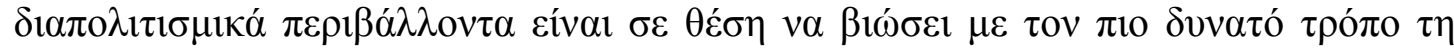

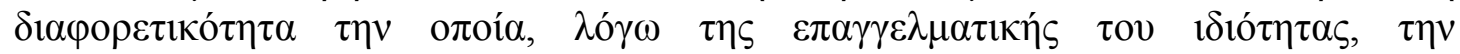




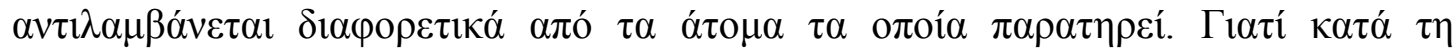

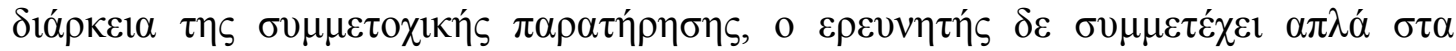

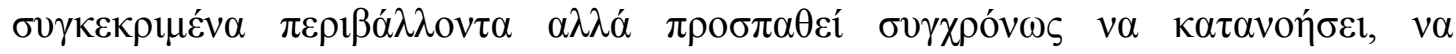

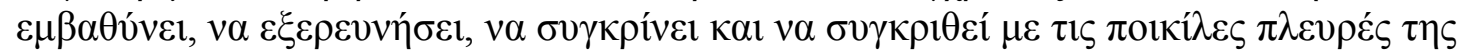

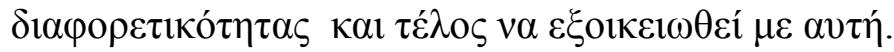

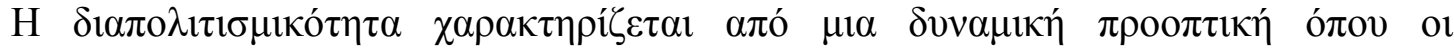

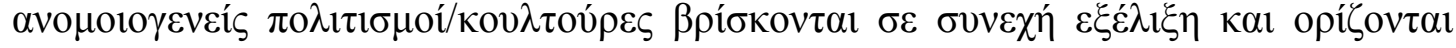

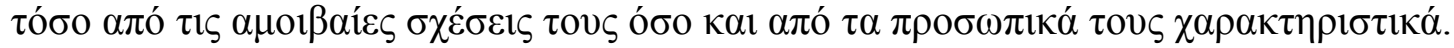

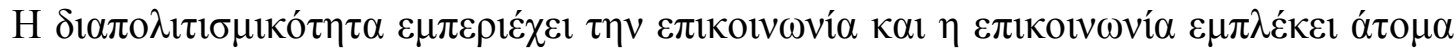

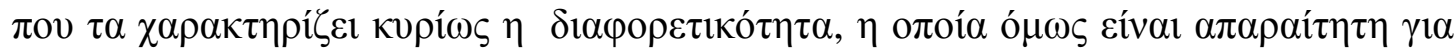

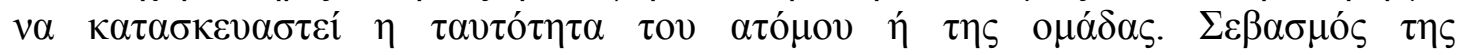

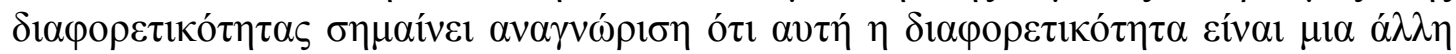

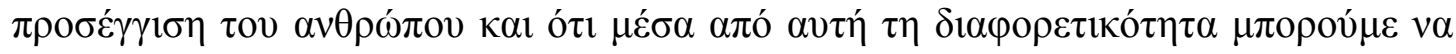

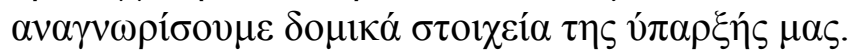

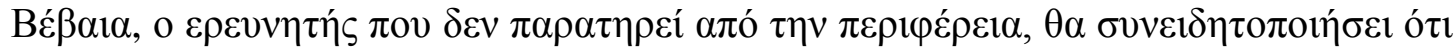

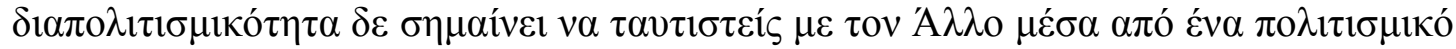

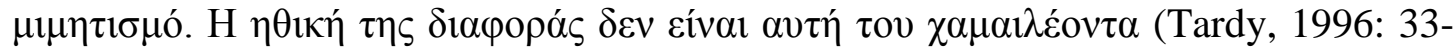

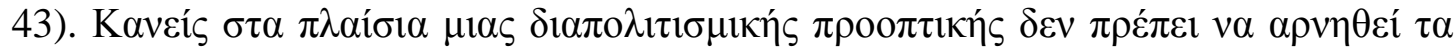

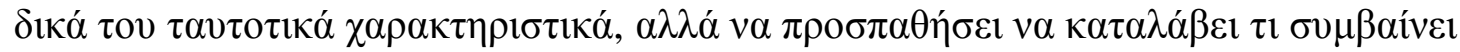

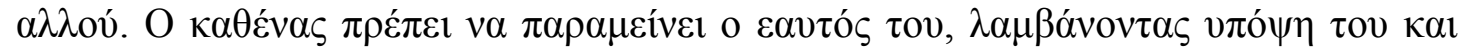

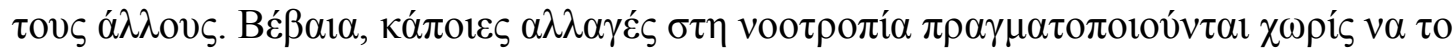

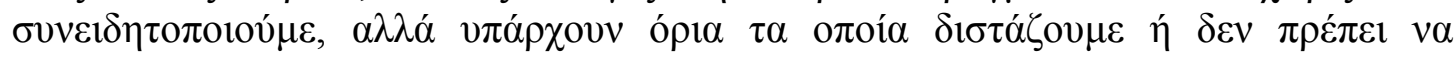

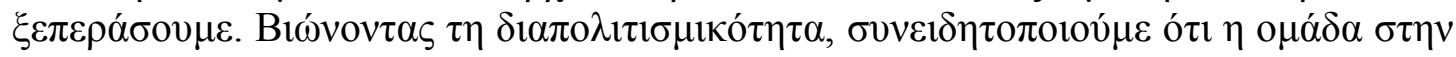

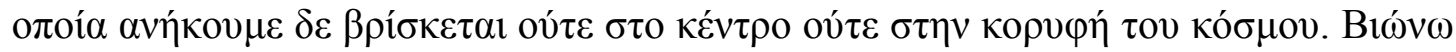

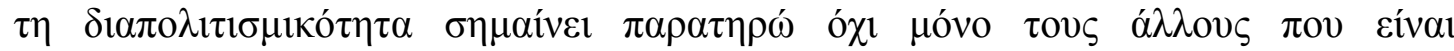

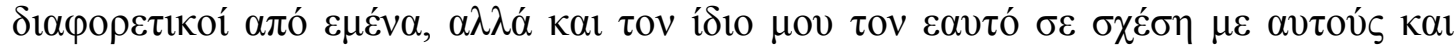

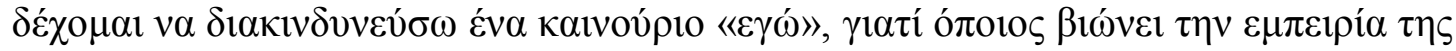

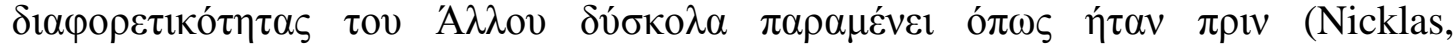
1996:177-187).

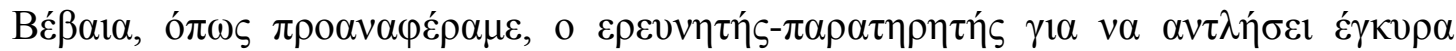

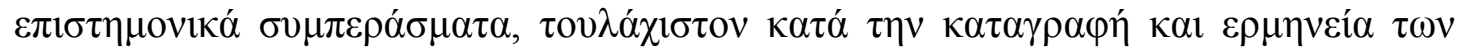

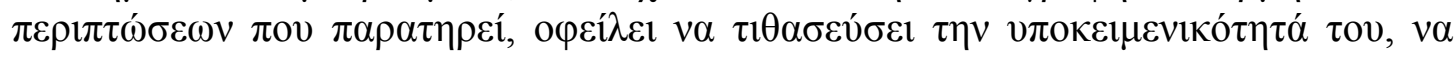

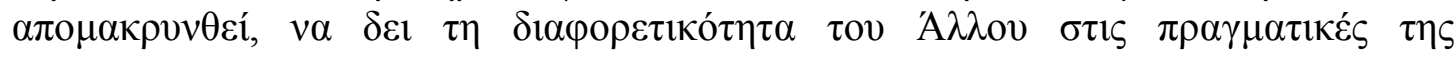

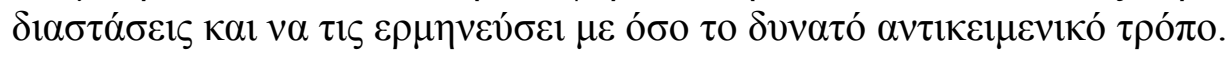

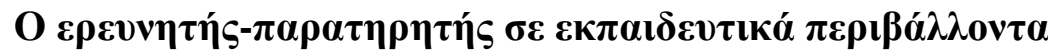

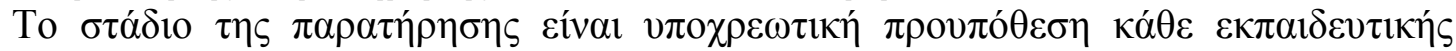

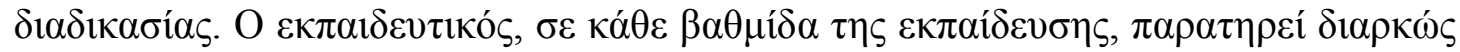

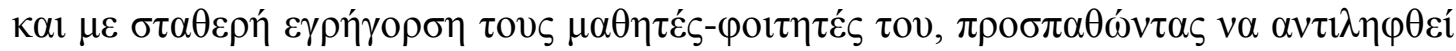

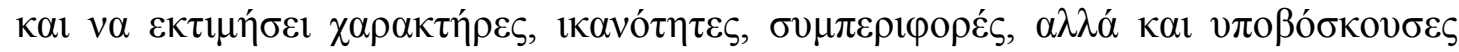

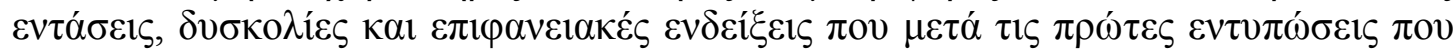

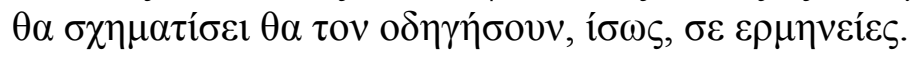

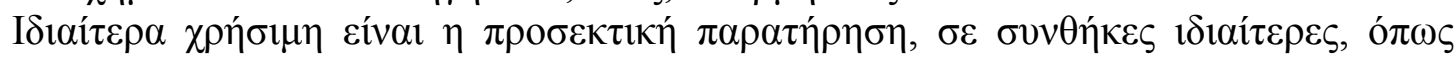

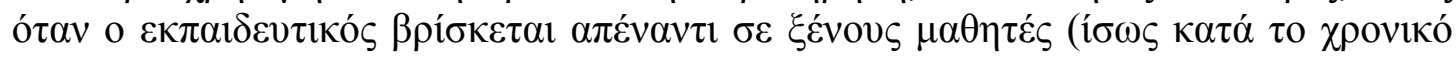

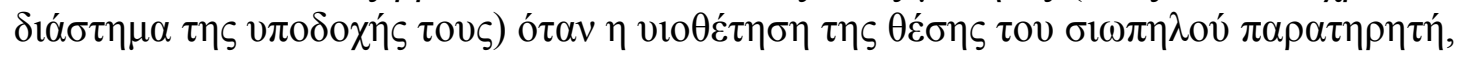

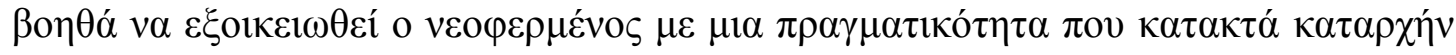

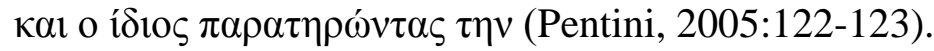

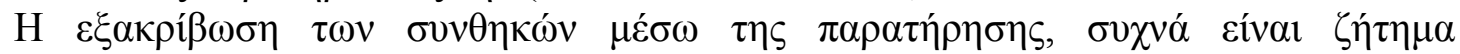

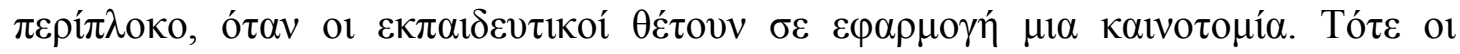

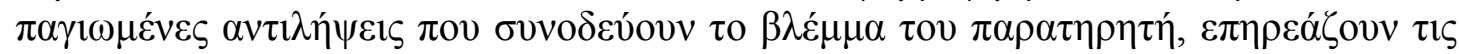




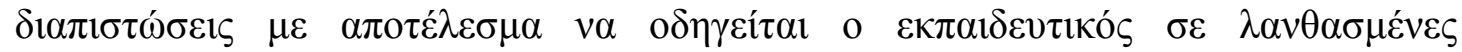

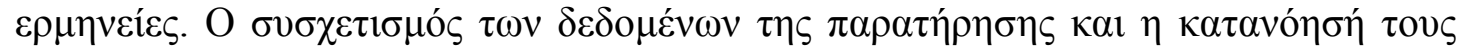

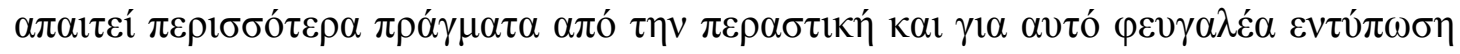
$\varepsilon v o ́ \varsigma \pi \alpha \rho \alpha \tau \eta \rho \eta \tau \eta ́, ~ \pi o v ~ \pi \rho \varepsilon ́ \pi \varepsilon 1 ~ \tau \alpha v \tau o ́ \chi \rho o v \alpha, ~ \varepsilon \varphi o ́ \sigma o v ~ \alpha v \alpha \varphi \varepsilon \rho o ́ \mu \alpha \sigma \tau \varepsilon ~ \sigma \varepsilon \varepsilon \kappa \pi \alpha 1 \delta \varepsilon v \tau 1 \kappa o u ́ \varsigma$,

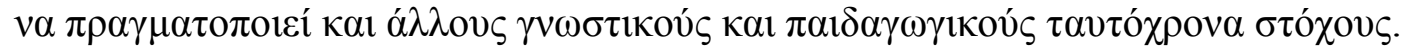

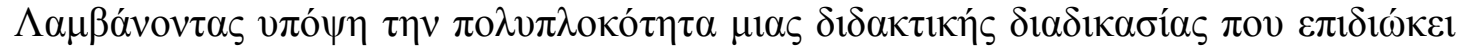

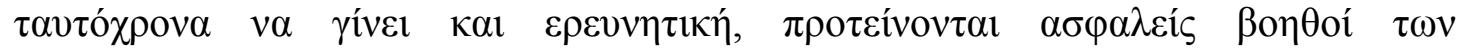

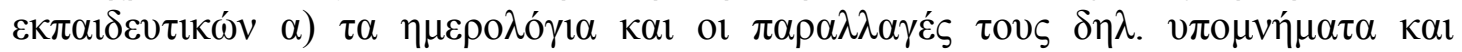

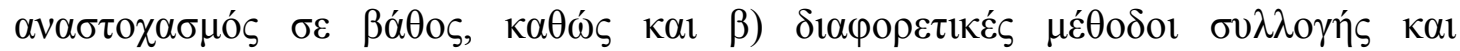

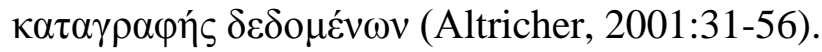

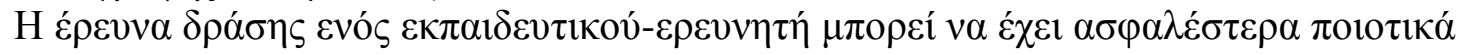

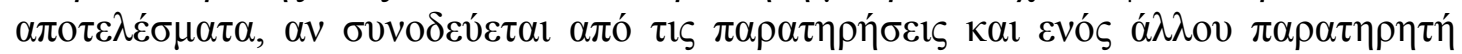

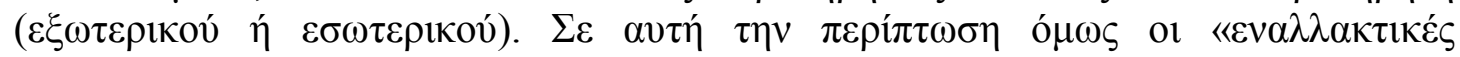

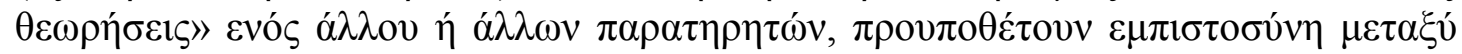

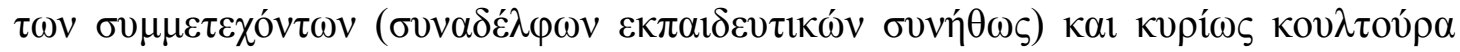

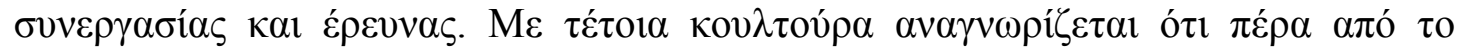

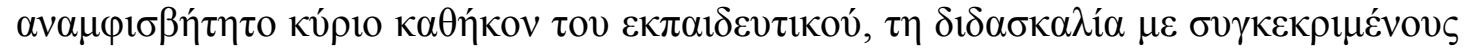

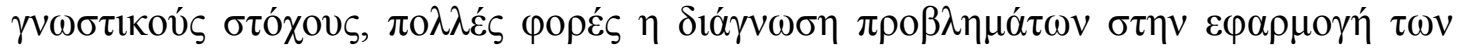

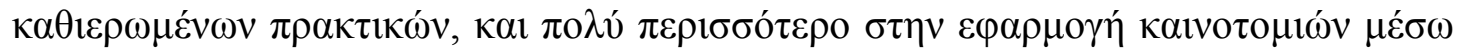

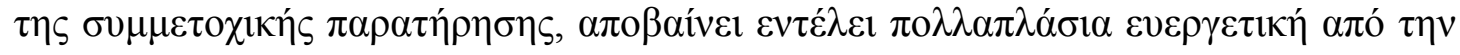

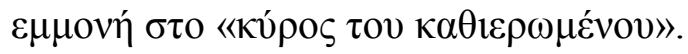

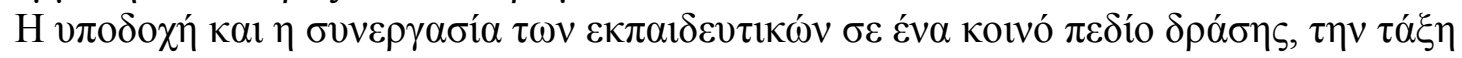

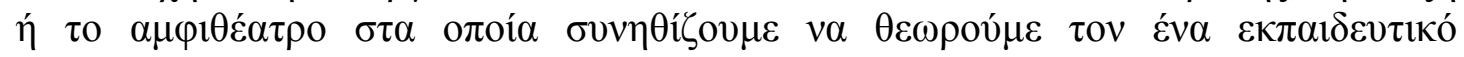

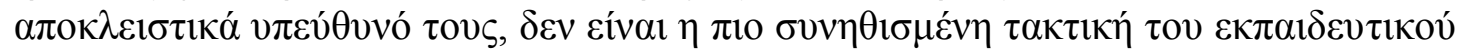

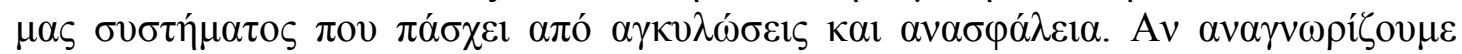

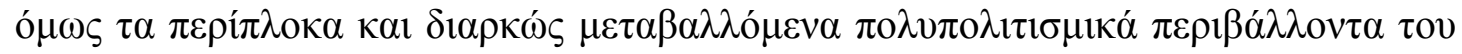

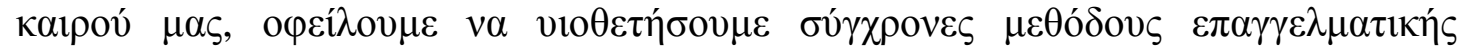

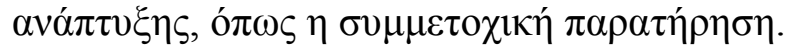

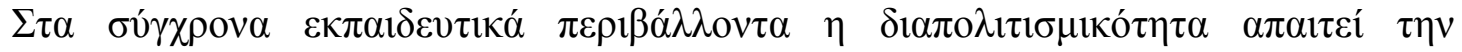

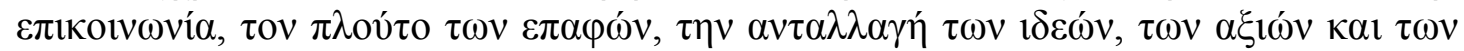

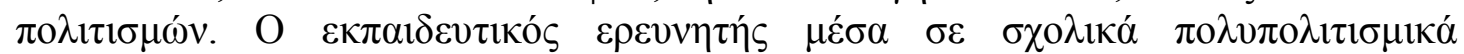

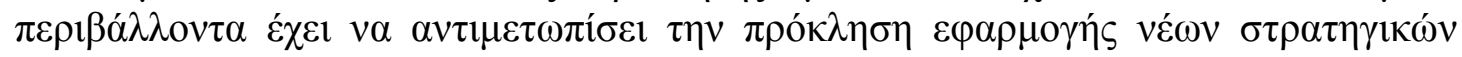

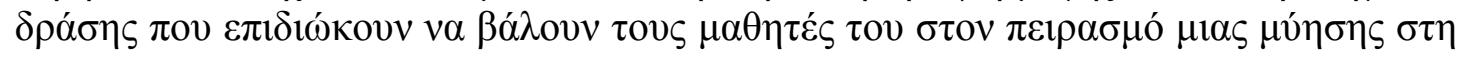

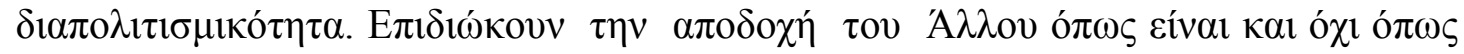

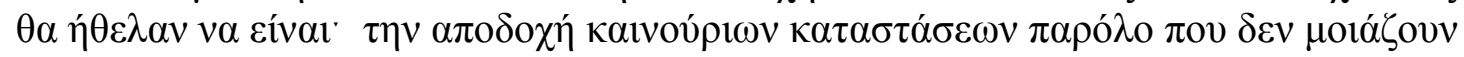

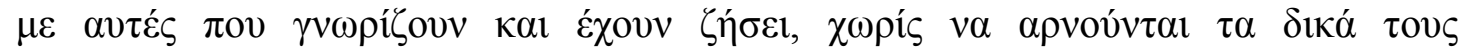

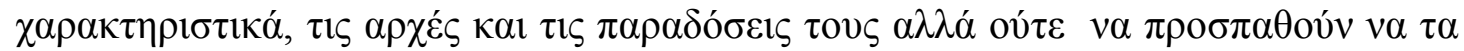

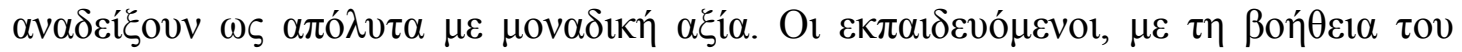

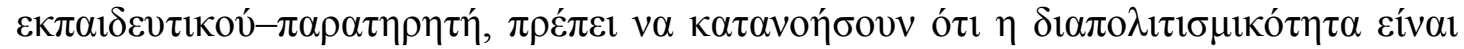

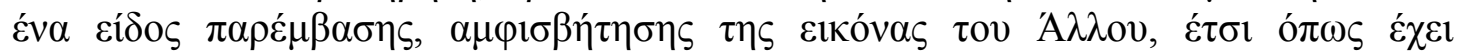

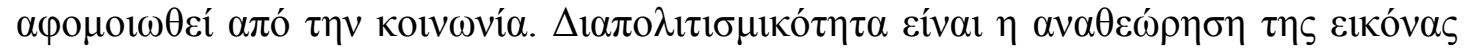

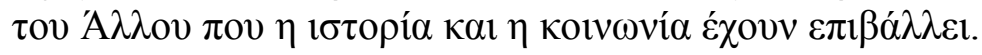

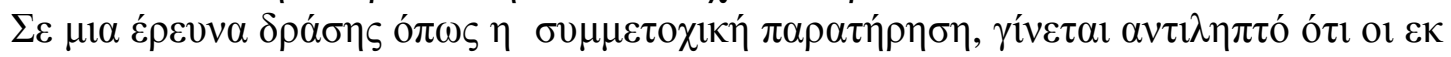

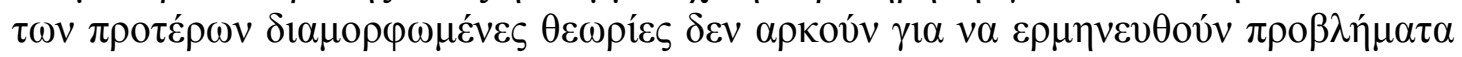

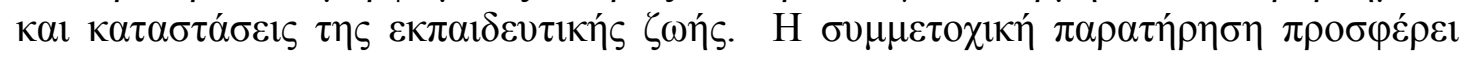

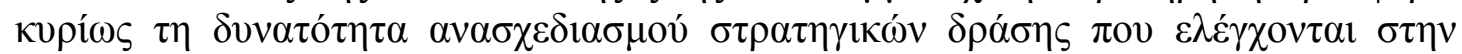

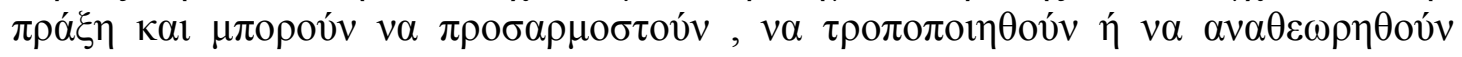

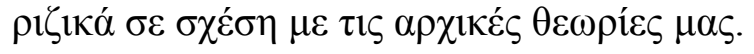




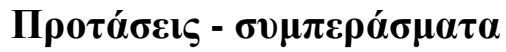

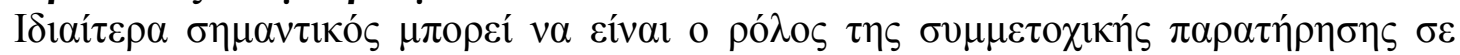

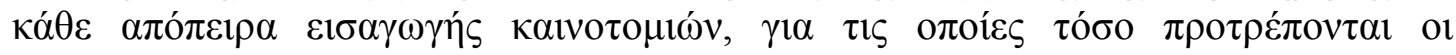

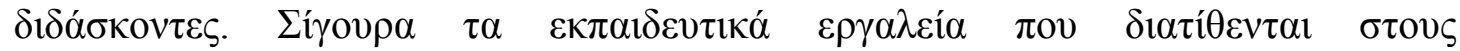

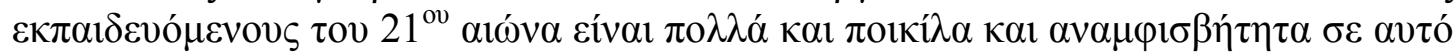

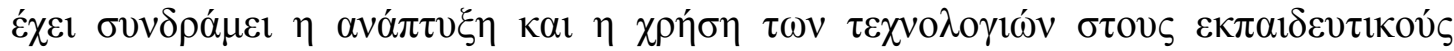

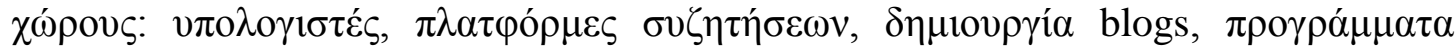

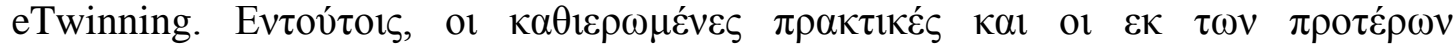

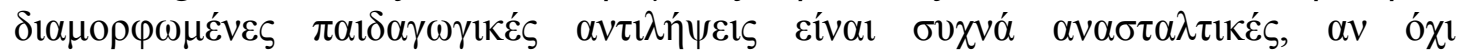

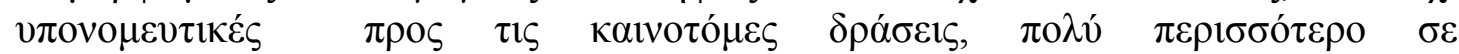

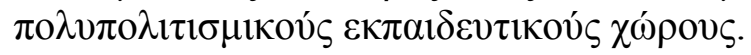

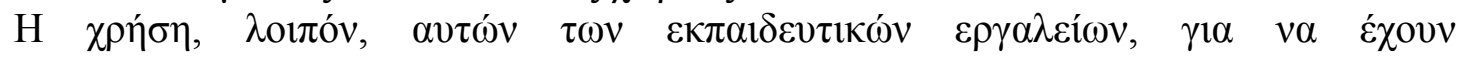

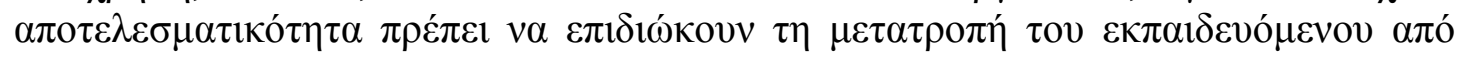

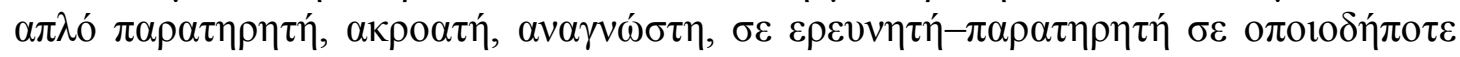

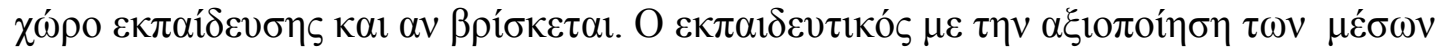

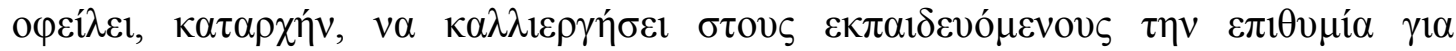

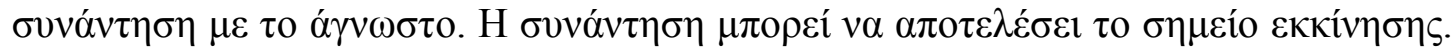

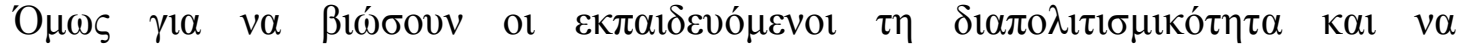

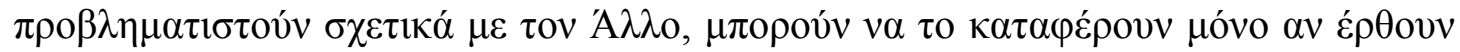

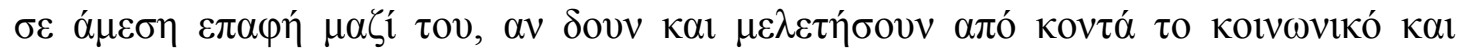

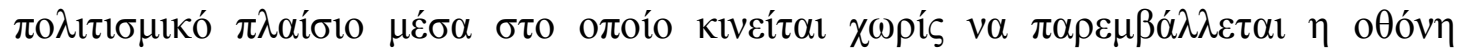

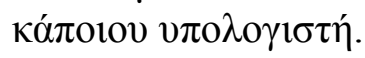

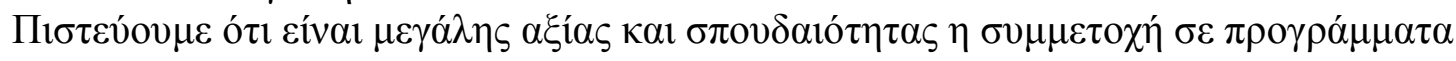

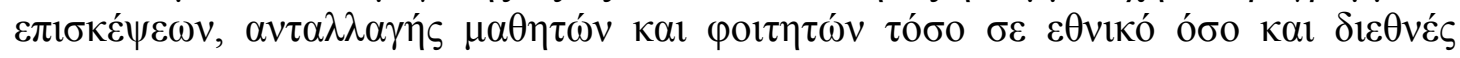

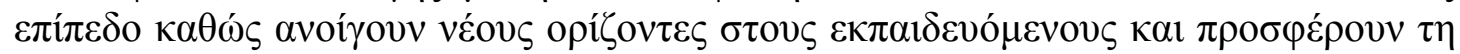

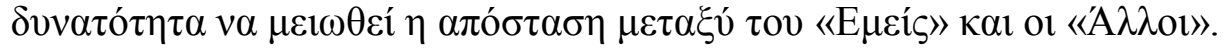

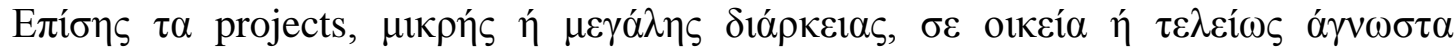

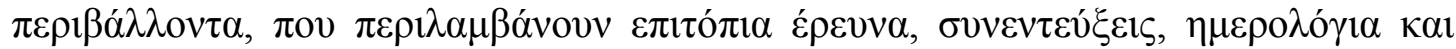

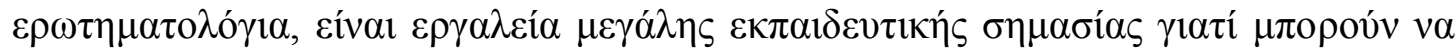

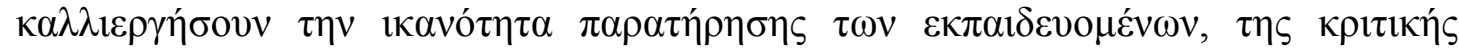

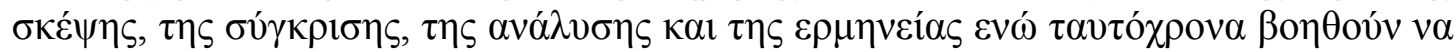

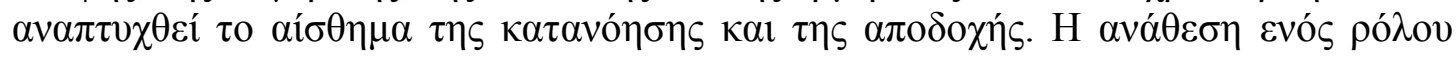

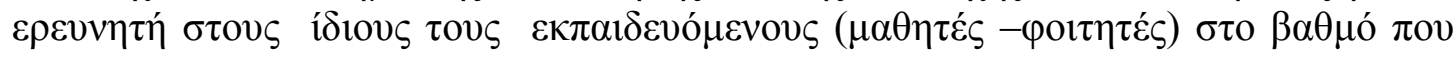

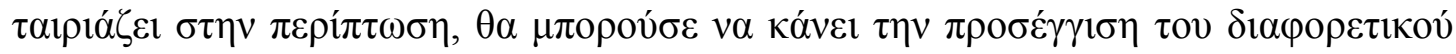

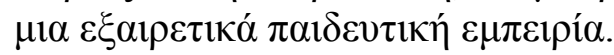

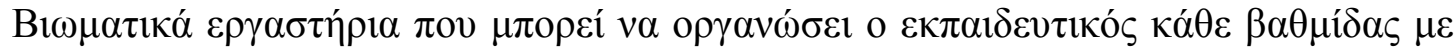

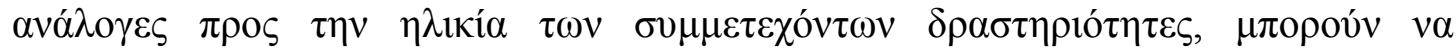

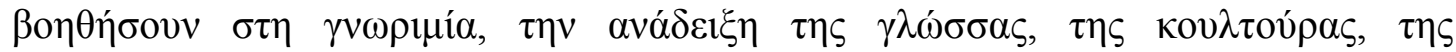

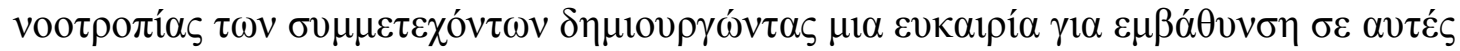
$\tau ı \zeta$ Évvoies.

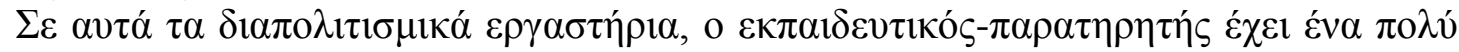

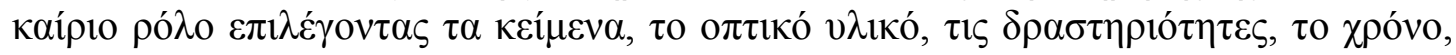

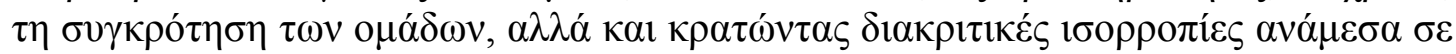

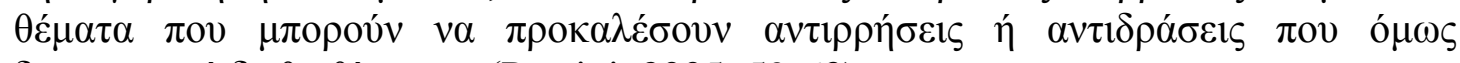

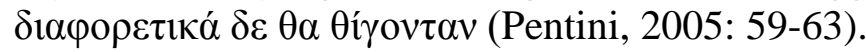

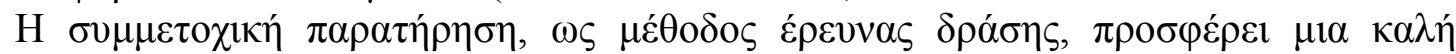

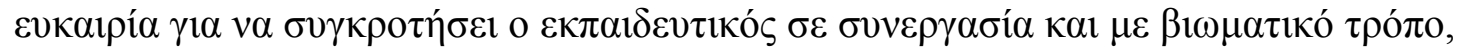

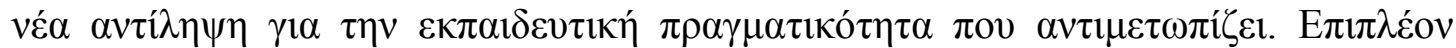

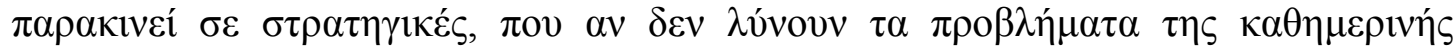




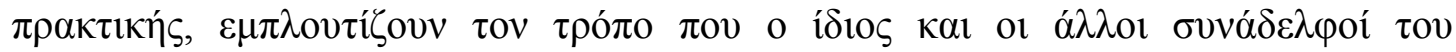

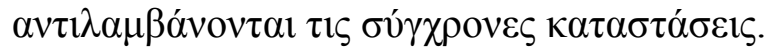

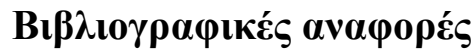

Adler, Patricia - Adler Peter (1987). Membership roles in field Research, USA, Sage Publications

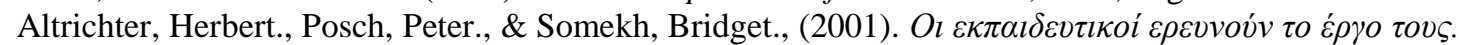

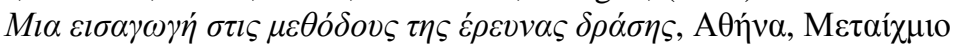

Blumer, Herbert (1969). Symbolic Interactionism, N. J., Prentice Hall

Booth, Charles (1902-1904). Life and labour of the people of London, London, McMillan \& Co

Chapoulie, Jean-Michel (1998). La place du terrain dans les sciences sociales, Le regard sociologique. EHESS

Coulon, André (1992). L'école de Chicago, Paris, PUF

Deliège, Robert (1994). Anthropologie sociale et culturelle. Bruxelles, De Boeck Université

Junker, Buford (1960). Fieldwork: an introduction to the Social Sciences, Chicago, The University of Chicago Press

Kellog, Paul (1909-1914). The Pittsburgh survey, New York, Russel Sage Foundation

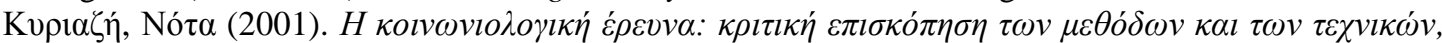

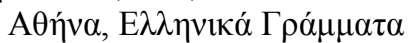

Lapassade, Georges (1991). L'ethnosociologie, Paris, Meridiens-Klingsieck

Lapassade, Georges (2006). L'observation participante, in Remi Hess - Weigand Gabriele, L'observation participante. Paris, ed. ECONOMICA, anthropos

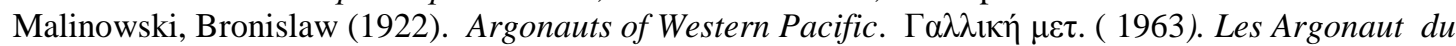
Pacifique occidental. Paris, Gallimard

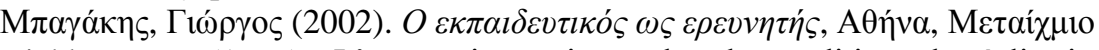

Nicklas, Hans (1996). L'apprentissage interculturel : conditions de réalisation et objectifs, in Lucette Colin et Burkhard Muller, La pédagogie des rencontres interculturelles. Paris, éd. anthropos

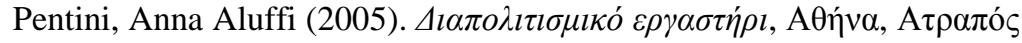

Tardy, Michel (1996). Education, socialisation, enculturation, in Lucette Colin et Burkhard Muller, La pédagogie des rencontres interculturelles. Paris, éd. anthropos

Whyte, William Foot (1996). Street Corner Society. La structure sociale d'un quartier américain, Paris, La Découverte 